\title{
Orbit-equivalent infinite permutation groups
}

\author{
D.C. Lockett · H.D. Macpherson
}

Received: 29 June 2012 / Accepted: 13 February 2013 / Published online: 26 February 2013

(C) Springer Science+Business Media New York 2013

\begin{abstract}
Let $G, H$ be closed permutation groups on an infinite set $X$, with $H$ a subgroup of $G$. It is shown that if $G$ and $H$ are orbit-equivalent, that is, have the same orbits on the collection of finite subsets of $X$, and $G$ is primitive but not 2-transitive, then $G=H$.
\end{abstract}

Keywords Primitive permutation group · Orbit-equivalent $\cdot$ Set-homogeneous

\section{Introduction}

We consider closed permutation groups acting on an infinite set $X$; that is, subgroups $G$ of $\operatorname{Sym}(X)$ which have the form $\operatorname{Aut}(M)$ for some relational structure $M$ with domain $X$. Two permutation groups $G, H$ on the set $X$ are said to be orbit-equivalent if, for every positive integer $k$, the groups $G$ and $H$ have the same orbits on the collection of unordered $k$-element subsets of $X$. This generalises a definition for finite permutation groups. Observe that if $G, H$ are orbit-equivalent, then they are each orbit-equivalent to $\langle G, H\rangle$. Thus, to investigate such pairs, it suffices (in part) to consider $G, H$ with $H$ a subgroup of $G$. Easily, if $G, H$ are orbit-equivalent, then $G$ is transitive (on $X$ ) if and only if $H$ is transitive, and also $G$ and $H$ preserve the same systems of imprimitivity on $X$; so $G$ is primitive on $X$ (that is, preserves no proper non-trivial equivalence relation on $X$ ) if and only if $H$ is primitive.

Our main theorem is the following. Our particular interest is in the case when $X$ is countably infinite, but the proofs below do not use countability.

Theorem 1.1 Let $G, H$ be orbit-equivalent closed permutation groups on the infinite set $X$, with $H \leq G$, and suppose that $G$ is primitive but not 2-transitive. Then $H=G$.

D.C. Lockett · H.D. Macpherson ( $₫$ )

School of Mathematics, University of Leeds, Leeds LS2 9JT, UK

e-mail: h.d.macpherson@leeds.ac.uk 
See Sect. 4 for a discussion of a possible strengthening, suggested by a referee, where the assumption $H \leq G$ is weakened, replaced by the assumption that $H$ is closed and not 2-transitive.

It is easily checked (see e.g. [3, Sect. 2.4]) that a subgroup of $\operatorname{Sym}(X)$ is closed in the sense defined above if and only if it is closed in $\operatorname{Sym}(X)$ in the topology of pointwise convergence on $\operatorname{Sym}(X)$ with respect to the discrete topology on $X$, where the basic open sets are cosets of pointwise stabilisers of finite sets; that is, basic open sets have the form $U_{f_{0}}:=\left\{f \in \operatorname{Sym}(X): f\right.$ extends $\left.f_{0}\right\}$ for some bijection $f_{0}$ between finite subsets of $X$. Thus, if $G \leq \operatorname{Sym}(X)$ is closed then $H \leq G$ is dense in $G$ if and only if $H$ and $G$ have the same orbits on $X^{k}$ for all $k>0$. In particular, if $H$ is a closed proper subgroup of $G \leq \operatorname{Sym}(X)$, then for some $k>0$, some $G$-orbit on $X^{k}$ (the set of $k$-tuples from $X$ ) breaks into more than one $H$-orbit. The assumption in the theorem that $G$ and $H$ are closed seems essential; indeed, any subgroup $H$ of $\operatorname{Sym}(X)$ is orbit-equivalent to its closure, and, for example, the dense (and so orbitequivalent) subgroups of $\operatorname{Sym}(X)$ are exactly the subgroups of $\operatorname{Sym}(X)$ which are $k$-transitive for all positive integers $k$, and these seem hopelessly unclassifiable.

This paper takes its motivation from two sources. First, there is an extended literature on primitive orbit-equivalent pairs of permutation groups on a finite set $X$; see for example $[12,21,22]$. Clearly, the symmetric and alternating groups $\mathrm{Sym}_{n}$ and $\mathrm{Alt}_{n}$, in their natural actions on $\{1, \ldots, n\}$, are orbit-equivalent for $n \geq 3$. Also, if $G$ is a permutation group on a finite set $X$ and has a regular orbit $U$ on the power set $\mathcal{P}(X)$, and $H$ is a proper subgroup of $G$, then $H$ is intransitive on $U$, and so $H$ is not orbit-equivalent to $G$. It is shown in [4] that if $X$ is finite then there are just finitely many primitive subgroups of $\operatorname{Sym}(X)$ which do not contain $\operatorname{Alt}(X)$ and have no regular orbit on $\mathcal{P}(X)$ (and so could have an orbit-equivalent proper subgroup). Such primitive groups $G$ (with no regular orbit on $X$ ) are classified by Seress in [19], who then classifies all pairs of finite primitive orbit-equivalent permutation groups $(H, G)$ with $H<G$. There is further work on the finite imprimitive case in [20].

Let us say that two permutation groups $G, H$ on a countably infinite set $X$ are strongly orbit-equivalent if they have the same orbits on the power set $\mathcal{P}(X)$ (not just on the collection of finite subsets of $X$ ). As pointed out by the referee, it is known that if $G, H$ are strongly orbit-equivalent permutation groups on the countably infinite set $X$, with $H \leq G$, and $G$ is primitive and each orbit of a point stabiliser $G_{X}$ is finite, then $H=G$; there is no topological closure assumption here. Indeed, by [24, Corollary 6], $G$ has a regular orbit on $\mathcal{P}(X)$, from which the assertion follows.

The second source of motivation is more model-theoretic, namely the study of homogeneous structures. Recall that a countable (possibly finite) structure $M$ in a first order relational language is said to be homogeneous if every isomorphism between finite substructures of $M$ extends to an automorphism of $M$. A natural generalisation, originally considered by Fraïssé in [9], is to say that the countable structure $M$ is set-homogeneous if, whenever $U, V$ are isomorphic finite substructures of $M$, there is $g \in \operatorname{Aut}(M)$ with $U^{g}=V$. Finite set-homogeneous graphs are classified by Ronse in [18], and a very short proof was given by Enomoto in [8] that every finite set-homogeneous graph is homogeneous. There is a classification of finite sethomogeneous digraphs (allowing two vertices to be linked by an arc in each direction) in [10], building on a corresponding classification of finite homogeneous digraphs by 
Lachlan [13]. Also, there are initial results on countably infinite set-homogeneous structures, in particular graphs and digraphs, in [7] and [10]. The latter paper poses the following related question: given a homogeneous structure $M$, when does $\operatorname{Aut}(M)$ have a proper closed subgroup $H$ which acts set-homogeneously on $M$, that is, has the same orbits as $\operatorname{Aut}(M)$ on the collection of unordered finite subsets of $M$ ? Equivalently, for which $M$ does $\operatorname{Aut}(M)$ have a proper closed orbit-equivalent subgroup? (Here, and throughout the paper, we use the same symbol $M$ for a structure and for its domain.)

A countably infinite set $X$ in the empty language is homogeneous, and has automorphism group $\operatorname{Sym}(X)$. By a theorem of Cameron [2], $\operatorname{Sym}(X)$ has just four orbit-equivalent closed proper subgroups, namely $\operatorname{Aut}(X,<), \operatorname{Aut}(X, B), \operatorname{Aut}(X, C)$, and $\operatorname{Aut}(X, S)$. Here $<$ is a dense linear order without end points on $X, B$ is the (ternary) linear betweenness relation on $X$ induced from $<, C$ is the (also ternary) circular order on $X$ induced from $<$, and $S$ is the corresponding arity 4 separation relation. Observe that $\operatorname{Aut}(X, S)=\langle\operatorname{Aut}(X, B), \operatorname{Aut}(X, C)\rangle$ and is 3-transitive but not 4-transitive. Our conjecture below would strengthen Theorem 1.1 in the countable case by removing the 'not 2-transitive' assumption.

Conjecture 1.2 Let $G$ and $H$ be distinct orbit-equivalent primitive closed permutation groups on a countably infinite set $X$. Then $G$ and $H$ belong to the list $\operatorname{Aut}(X,<)$, $\operatorname{Aut}(X, B), \operatorname{Aut}(X, C), \operatorname{Aut}(X, S), \operatorname{Sym}(X)$ described above.

Recall the following standard terminology, for a permutation group $G$ on a set $X$, and an integer $k>0$ : $G$ is $k$-transitive if it is transitive on the ordered $k$-subsets of $X$; and $G$ is $k$-homogeneous if it is transitive on the unordered $k$-subsets of $X$. Also, if $U$ is a subset of $X$ then $G_{\{U\}}$ and $G_{(U)}$ denote, respectively, the setwise and pointwise stabilisers of $U$ in $G$, and if $x \in X$ then $G_{x}:=\left\{g \in G: x^{g}=x\right\}$.

The proof of Theorem 1.1 splits into two cases:

(1) $G$ is primitive but not 2-homogeneous;

(2) $G$ is 2-homogeneous (and so primitive) but is not 2-transitive.

Our main tool for both cases is the notion of local rigidity, introduced, so far as we know, in [7]. We shall say that a permutation group $G$ acting on an infinite set $X$ acts locally rigidly if for all finite $U \subset X$, there is some finite $V \subset X$ such that $U \subseteq V$ and the setwise stabiliser $G_{\{V\}}$ of $V$ fixes $U$ pointwise. Likewise, a first order relational structure $M$ is locally rigid if, for every finite substructure $U$ of $M$, there is a finite substructure $V$ of $M$ containing $U$ such that every automorphism of $V$ fixes $U$ pointwise. Clearly, if a relational structure $M$ is locally rigid, then any subgroup of its automorphism group acts locally rigidly on $M$. Strengthening the notion of local rigidity, we shall later say that an infinite first order structure $M$ is cofinally rigid if, for every finite substructure $U$ of $M$, there is a finite substructure $V$ of $M$ with $U \subseteq V$ such that the automorphism group of $V$ is trivial. Here, 'substructure' is used in the standard model-theoretic sense, corresponding to the graph-theoretic notion of 'induced subgraph'.

Lemma 1.3 Let $G, H$ be closed permutation groups on $X$, with $H \leq G$. If $G$ and $H$ are orbit-equivalent and $G$ acts locally rigidly, then $H=G$. 
Proof It suffices to show that $H$ has the same orbits as $G$ on $X^{k}$ for all $k$. So let $\bar{u}_{1}, \bar{u}_{2} \in X^{k}$ be in the same orbit of $G$; that is, there is $g \in G$ such that $\bar{u}_{1}^{g}=\bar{u}_{2}$. Let $U_{1}, U_{2} \subset X$ be enumerated by $\bar{u}_{1}, \bar{u}_{2}$, respectively. Since $G$ acts locally rigidly on $X$, there is finite $V_{1} \subset X$ such that $U_{1} \subseteq V_{1}$ and $G_{\left\{V_{1}\right\}} \leq G_{\left(U_{1}\right)}$. Let $V_{2}:=V_{1}^{g}$. Then $V_{1}, V_{2}$ are in the same orbit of $G$ on $\left|V_{1}\right|$-sets, so by orbit-equivalence there is some $h \in H$ such that $V_{1}^{h}=V_{2}$. Now $g h^{-1} \in G_{\left\{V_{1}\right\}}$, so in fact $g h^{-1} \in G_{\left(U_{1}\right)}$. Thus $\bar{u}_{1}^{h}=\bar{u}_{2}$ as required.

In both cases (1) and (2) ( $G$ primitive, and either not 2-homogeneous, or 2homogeneous but not 2-transitive) we shall show that $G$ acts locally rigidly on $X$. In fact, in the second case we show that $G$ is a group of automorphisms of a cofinally rigid tournament. Our method to show the local rigidity of such actions stems from a similar result in [7], which we adapt. Formally, we view a graph $\Gamma$ as a relational structure $\Gamma=(X, R)$, where $R$ is a symmetric irreflexive binary relation on $X$. Given a graph $\Gamma$, if $x, y$ are vertices we write $x \sim y$ if $x$ and $y$ are adjacent, and let $\Gamma(x):=\{v \in X: v \sim x\}$, the neighbour set of $x$. We shall prove in Lemma 2.3 a strengthening of the following result.

Lemma 1.4 [7] Let $\Gamma$ be an infinite graph such that, for all distinct vertices $x, y$ of $\Gamma$, the sets $\Gamma(x) \backslash \Gamma(y)$ and $\Gamma(y) \backslash \Gamma(x)$ are both infinite. Then $\Gamma$ is locally rigid.

We draw attention to a basic Ramsey-theoretic principle which is well-known, for example in model theory, and used below in both the primitive not 2-homogeneous case, and the 2-homogeneous not 2-transitive case.

Definition 1.5 Let $L$ be a finite relational language, let $M$ be a first order $L$-structure, $A$ a finite subset of the domain of $M$, and $P_{1}, \ldots, P_{r}$ disjoint subsets of $M \backslash A$, with $P_{i}:=\left\{p_{i, 0}, \ldots, p_{i, n-1}\right\}$ for each $i=1, \ldots, r$. We say that $P_{1} \ldots, P_{r}$ are mutually indiscernible over $A$ if the following holds for any non-negative integers $e_{1}, \ldots, e_{r}<$ $n$ : for each $j=1, \ldots, r$, let $\bar{p}_{j}, \bar{p}_{j}^{\prime}$ be $e_{j}$-tuples from $P_{j}$, each listed in increasing order (so if $\bar{p}_{j}=\left(p_{j, i(1)}, \ldots, p_{j, i\left(e_{j}\right)}\right)$, then $\left.i(1)<\cdots<i\left(e_{j}\right)\right)$; then the map taking $\bar{p}_{j}$ to $\bar{p}_{j}^{\prime}$ for each $j$, extended by the identity on $A$, is an isomorphism of $L$-structures.

As a small example, let $L$ consist of binary relations $R$ and $<$, let $A=\left\{a_{1}, a_{2}\right\}$, $P_{1}=\left\{p_{1 i}: i \in \mathbb{N}\right\}$, and $P_{2}=\left\{p_{2 i}: i \in \mathbb{N}\right\}$, with $a_{1}, a_{2}$ and the $p_{j i}$ all distinct, and let $M \supset A \cup P_{1} \cup P_{2}$. Suppose that $R$ is symmetric and irreflexive, so determines a graph structure on $M$, with $P_{1}$ a complete subgraph with all its elements adjacent to $a_{1}$, and with no other adjacencies on $A \cup P_{1} \cup P_{2}$. Suppose also that $p_{1 i}<p_{1 j}$ whenever $i, j \in \mathbb{N}$ with $i<j$, and that $<$ does not hold between other pairs from $A \cup P_{1} \cup P_{2}$. Then $P_{1}, P_{2}$ are mutually indiscernible over $A$.

Lemma 1.6 Let $M, L, A$ be as in Definition 1.5 with $M$ infinite, and let $Q_{1}, \ldots, Q_{r}$ be countably infinite disjoint subsets of $M \backslash A$. Let $n$ be a positive integer. Then the following hold.

(i) There are subsets $P_{1} \subset Q_{1}, \ldots, P_{r} \subset Q_{r}$, each of size $n$, such that $P_{1}, \ldots, P_{r}$ are mutually indiscernible over $A$ (with respect to some indexing of each $P_{i}$ ). 
(ii) If every relation of $L$ is of arity at most 2 , and $P_{1}, \ldots, P_{r}$ are as in $(i)$, then for each $i=1, \ldots, r$, either some relation of $L$ induces a total order on $P_{i}$, or every permutation of $P_{i}$, extended by the identity on $R_{i}:=A \cup \bigcup_{j \neq i} P_{j}$, is an automorphism of the induced $L$-structure on $R:=A \cup P_{1} \cup \cdots \cup P_{r}$.

Proof (i) Let $Q_{i}:=\left\{q_{i, j}: j \in \mathbb{N}\right\}$ for each $i=1, \ldots, r$. Let $d$ be the maximum arity of a relation in $L$. Colour each subset $\left\{i_{1}, \ldots, i_{r d}\right\}$ of $\mathbb{N}$ in such a way that given natural numbers $i_{1}<\cdots<i_{r d}$ and $k_{1}<\cdots<k_{r d}$, the map

$$
\left(q_{1, i_{1}}, \ldots, q_{1, i_{r d}}, \ldots, q_{r, i_{1}}, \ldots, q_{r, i_{r d}}\right) \mapsto\left(q_{1, k_{1}}, \ldots, q_{1, k_{r d}}, \ldots, q_{r, k_{1}}, \ldots, q_{r, k_{r d}}\right)
$$

is an isomorphism over $A$ if and only if $\left\{i_{1}, \ldots, i_{r d}\right\}$ and $\left\{k_{1}, \ldots, k_{r d}\right\}$ have the same colour. Finitely many colours are required; for since $L$ is finite, there are finitely many possible isomorphism types of tuple of length $r^{2} d+|A|$. By Ramsey's Theorem, replacing $\mathbb{N}$ by an infinite monochromatic subset if necessary, we may suppose that $\mathbb{N}$ is monochromatic. Now let $p_{i, j}:=q_{i,(i-1)(n+d)+j}$ for each $i=1, \ldots, r$ and $j=$ $0, \ldots, n+d-1$. Put $P_{i}^{\prime}:=\left\{p_{i, 0}, \ldots, p_{i, n+d-1}\right\}$ and $P_{i}:=\left\{p_{i, 0}, \ldots, p_{i, n-1}\right\}$ for each $i=1, \ldots, r$.

We claim that $P_{1}, \ldots, P_{r}$ are mutually indiscernible over $A$. Indeed, let $e_{1}, \ldots, e_{r}$ be integers with $0 \leq e_{j}<n$ for each $n$, and for each $j$ let $\bar{p}_{j}$ and $\bar{p}_{j}^{\prime}$ be $e_{j}$-tuples from $P_{j}$, with $\bar{p}_{j}=\left(p_{j, i_{j}(1)}, \ldots, p_{j, i_{j}\left(e_{j}\right)}\right)$ for $0 \leq i_{j}(1)<\cdots<i_{j}\left(e_{j}\right)<n$, and $\bar{p}_{j}^{\prime}=\left(p_{j, i_{j}(1)^{\prime}}^{\prime}, \ldots, p_{j, i_{j}\left(e_{j}\right)^{\prime}}^{\prime}\right)$ for $0 \leq i_{j}(1)^{\prime}<\cdots<i_{j}\left(e_{j}\right)^{\prime}<n$. We must show that the map fixing $A$ pointwise and taking each $\bar{p}_{j}$ to $\bar{p}_{j}^{\prime}$ is an isomorphism. Since the language has arity $d$, we may suppose that each $\bar{p}_{j}$ has length at most $d$. Hence, by extending each $\bar{p}_{j}$ and $\bar{p}_{j}^{\prime}$ by entries from $P_{j}^{\prime} \backslash P_{j}$ if necessary, we may suppose that each $\bar{p}_{j}$ has length exactly $d$, that is, $e_{j}=e_{j}^{\prime}=d$ for each $j=1, \ldots, r$. If we now write $\bar{p}_{1} \ldots \bar{p}_{r}$ as a tuple of $q_{i j}$, then the second indices are increasing: formally,

$$
\bar{p}_{1} \ldots \bar{p}_{r}=\left(q_{1, i_{1}(1)}, \ldots, q_{1, i_{1}(d)}, \ldots, q_{r,(r-1)(n+d)+i_{r}(1)}, \ldots, q_{r,(r-1)(n+d)+i_{r}(d)}\right),
$$

with $i_{1}(1)<\cdots<i_{1}(d)<\cdots<(r-1)(n+d)+i_{r}(1)<\cdots<(r-1)(n+d)+i_{r}(d)$. The same holds for $\bar{p}_{1}^{\prime} \ldots \bar{p}_{r}^{\prime}$. It follows from the definition of the colouring that the map $\bar{p}_{1} \ldots \bar{p}_{r} \rightarrow \bar{p}_{1}^{\prime} \ldots \bar{p}_{r}^{\prime}$ is indeed an isomorphism over $A$.

(ii) This is immediate from (i). Indeed, suppose that no binary relation of $L$ induces a total order on $P_{i}$. Then by the indiscernibility (applied to subsets of $P_{i}$ of size at most 3 ) each binary relation is symmetric on $P_{i}$. It follows that any transposition $\left(p_{i, j}, p_{i, j+1}\right)$, extended by the identity on the rest of $R$, is an automorphism of $R$. Since such permutations generate a group fixing $R_{i}$ pointwise and inducing the symmetric group on $P_{i}$, the result follows.

The case of Theorem 1.1 when $G$ is primitive but not 2-homogeneous is handled in Sect. 2, and the 2-homogeneous but not 2-transitive case is treated in Sect. 3. Section 4 consists of some further observations, about bounds in local rigidity, approaches to Conjecture 1.2, and regular orbits on the power set. We also observe that our proofs give a slight strengthening of Theorem 1.1, namely Theorem 4.1. 


\section{$2 G$ primitive but not 2-homogeneous}

In this section we prove the following.

Proposition 2.1 Let $G$ be a primitive but not 2-homogeneous permutation group on an infinite set $X$. Then the action of $G$ on $X$ is locally rigid.

The proposition follows rapidly from the following two lemmas. The first uses an argument in [16, Proposition 4.4].

Lemma 2.2 Let $G$ be a primitive but not 2-homogeneous permutation group on an infinite set $X$. Then there is a $G$-invariant graph $\Gamma$ with vertex set $X$ such that for all distinct $x, y \in X$, the symmetric difference $\Gamma(x) \triangle \Gamma(y)$ is infinite.

Proof Let $U$ be any $G$-orbit on the collection of 2-subsets of $X$. Then $U$ is the edge set of a $G$-invariant graph $\Gamma_{0}$ with vertex set $X$, and as $G$ is not 2-homogeneous, $\Gamma_{0}$ is not complete. For $x \in X$, write $\Gamma_{0}(x)$ for the neighbour set of $x$ in $\Gamma_{0}$. Define the equivalence relation $\equiv_{0}$ on $X$, putting $x \equiv_{0} y$ if and only if $\left|\Gamma_{0}(x) \triangle \Gamma_{0}(y)\right|$ is finite. Then $\equiv_{0}$ is $G$-invariant, so by primitivity $\equiv_{0}$ is trivial or universal. The lemma holds if $\equiv_{0}$ is trivial, so we shall suppose that $\equiv_{0}$ is universal.

Recall that a graph is locally finite if all of its vertices have finite degree.

Claim Either $\Gamma_{0}$ or its complement is locally finite.

Proof of Claim Suppose not, and fix $x \in X$. Then both $\Gamma_{0}(x)$ and $X \backslash \Gamma_{0}(x)$ are infinite. If $y \in \Gamma_{0}(x)$ then (as $\equiv_{0}$ is universal) $\Gamma_{0}(y) \backslash \Gamma_{0}(x)$ is finite. Hence as $G_{x}$ has at most two orbits on $\Gamma_{0}(x)$ there is $k \in \mathbb{N}$ such that for all $y \in \Gamma_{0}(x)$, we have $\left|\Gamma_{0}(y) \backslash \Gamma_{0}(x)\right| \leq k$. Pick distinct $z_{1}, \ldots, z_{k+1} \in X \backslash\left(\{x\} \cup \Gamma_{0}(x)\right)$. Then as $x \equiv_{0} z_{i}$ for each $i$, each set $\Gamma_{0}\left(z_{i}\right) \cap \Gamma_{0}(x)$ is cofinite in $\Gamma_{0}(x)$. Hence there is $y \in \Gamma_{0}(x) \cap$ $\bigcap_{i=1}^{k+1} \Gamma_{0}\left(z_{i}\right)$. Then $z_{1}, \ldots, z_{k+1} \in \Gamma_{0}(y) \backslash \Gamma_{0}(x)$, so $\left|\Gamma_{0}(y) \backslash \Gamma_{0}(x)\right| \geq k+1$, which is a contradiction.

By the claim, replacing $\Gamma_{0}$ by its complement if necessary, we may suppose that $\Gamma_{0}$ is locally finite. By our original assumption that $\Gamma_{0}$ is not complete or null (that is, an independent set), $\Gamma_{0}$ has an edge. By primitivity, $\Gamma_{0}$ is connected. Now let $\Gamma$ be the graph on $X$ whose edge set consists of the set of unordered pairs an even distance apart in $\Gamma_{0}$. Then $\Gamma$ is also $G$-invariant. Pick $v_{0} \in X$, and choose a $\Gamma_{0}$ path $v_{0} \sim v_{1} \sim v_{2} \sim \ldots$ so that the distance $d_{0}\left(v_{0}, v_{i}\right)$ between $v_{0}$ and $v_{i}$ in $\Gamma_{0}$ equals $i$ for each $i$ (this is certainly possible, for example by König's Lemma). Then $v_{2 i} \in \Gamma\left(v_{0}\right) \backslash \Gamma\left(v_{1}\right)$ for each $i>0$. Thus $\Gamma\left(v_{0}\right)$ and $\Gamma\left(v_{1}\right)$ have infinite symmetric difference. It follows that $\equiv$ is not universal, where $\equiv$ is the $G$-invariant equivalence relation on $X$ defined by putting $x \equiv y$ whenever $\Gamma(x) \triangle \Gamma(y)$ is finite. Since $G$ is primitive, $\equiv$ is trivial, so $\Gamma(x) \triangle \Gamma(y)$ is infinite for all distinct $x, y \in X$.

In the next lemma, and later in the paper, if $A, B$ are sets we write $A \subset_{f} B$ if $B \backslash A$ is infinite and $A \backslash B$ is finite. The lemma below extends Lemma 1.4, since under 
the assumptions of that lemma, $x<y$ (as defined below) never holds. If $u, v, w$ are distinct vertices of the graph $\Gamma$, we say $w$ separates $u$ and $v$ if $w \in(\Gamma(u) \triangle \Gamma(v)) \backslash$ $\{u, v\}$, and call a collection of such vertices $w$ a separating set for $u$ and $v$.

Lemma 2.3 Let $\Gamma=(X, R)$ be an infinite graph, and suppose that $\Gamma(x) \triangle \Gamma(y)$ is infinite for any distinct $x, y \in X$. Write $x<y$ whenever $\Gamma(y) \subset_{f} \Gamma(x)$. Then the structure $\Gamma_{<}=(X, R,<)$ is locally rigid.

Proof We adapt the proof of Proposition 6.1 from [7]. So let $U=\left\{u_{1}, \ldots, u_{n}\right\}$ be a finite subset of $X$. We aim to find finite $V$ with $U \subset V \subset X$, such that $\operatorname{Aut}(V, R,<)$ fixes $U$ pointwise.

For each $u_{i}, u_{j} \in U$, with $i<j$, we find an infinite separating set $Q_{i j} \subset X \backslash U$ as follows: if $u_{i}<u_{j}$, then let $Q_{i j} \subset \Gamma\left(u_{i}\right) \backslash\left(\Gamma\left(u_{j}\right) \cup\left\{u_{j}\right\}\right)$; if $u_{j}<u_{i}$, then let $Q_{i j} \subset \Gamma\left(u_{j}\right) \backslash\left(\Gamma\left(u_{i}\right) \cup\left\{u_{i}\right\}\right)$; and if $u_{i} \| u_{j}$ (that is, $u_{i}, u_{j}$ are incomparable under $<)$, then let $Q_{i j} \subset \Gamma\left(u_{i}\right) \backslash\left(\Gamma\left(u_{j}\right) \cup\left\{u_{j}\right\}\right)$. By refining the $Q_{i j}$ further, we may suppose that if $i<j$ and $k<l$ with $(i, j) \neq(k, l)$, then $Q_{i j} \cap Q_{k l}=\emptyset$.

Let $K$ be a positive integer, chosen sufficiently large for the argument below. By Lemma 1.6 with respect to the language $L=\{R,<\}$, we can choose for each $i<j$ a subset $P_{i j}$ of $Q_{i j}$ with $\left|P_{i j}\right|=K$, such that the collection of all sets $P_{i j}$ is mutually indiscernible over $U$. Let $W=U \cup \bigcup\left(P_{i j}: 1 \leq i<j \leq n\right)$. Then each $P_{i j}$ carries a complete or null induced graph structure, and for each $x, y \in P_{i j}$ and $z \in W \backslash P_{i j}$, we have $x \sim z$ if and only if $y \sim z$.

For any subset $Y$ of $X$, define the equivalence relation $\approx_{Y}$ on $Y$, where, for $x, y \in$ $Y, x \approx_{Y} y$ if and only if $(\Gamma(x) \triangle \Gamma(y)) \cap Y \subseteq\{x, y\}$. Then $\approx_{Y}$-classes always carry a complete or null induced subgraph structure. If $Z$ is an $\approx_{Y}$-class, then for $z_{1}, z_{2} \in Z$ and $y \in Y \backslash Z$, we have $y \sim z_{1} \Leftrightarrow y \sim z_{2}$; in particular, all elements of $\operatorname{Sym}(Y)_{(Y \backslash Z)}$ induce automorphisms of $(Y, R)$. Observe that if $Y_{1} \subset Y_{2} \subseteq X$ and $x, y \in Y_{1}$, then $x \approx_{Y_{2}} y$ implies $x \approx_{Y_{1}} y$. We often identify such $Y$ with the induced subgraph $(Y, R \cap$ $\left.Y^{2}\right)$ of $\Gamma$ which it carries. Thus, $\approx_{Y}$ is $\operatorname{Aut}(Y, R)$-invariant.

Now, each $P_{i j}$ lies in a $\approx_{W}$-class of $W$. Deleting some sets $P_{i j}$ if necessary (only where elements of distinct sets $P_{i j}$ are $\approx_{W}$-equivalent, and retaining the assumption that any two distinct elements of $U$ are separated by some set of form $P_{i j}$ ), we may suppose: no two elements $x, y$ in distinct sets $P_{i j}, P_{k l}$ are $\approx_{W}$-equivalent. Also, $\approx_{W^{-}}$ classes contain at most one point of $U$; for if $u_{i}, u_{j} \in U$ with $i<j$ then there is a non-empty set $P_{k l}$ whose elements separate $u_{i}$ and $u_{j}$, so witness that $u_{i} \approx_{W} u_{j}$. Let $m=\left(\begin{array}{c}n \\ 2\end{array}\right)$, an upper bound on the number of distinct sets $P_{i j}$. Adjusting the $P_{i j}$ and hence $W$ further, we arrange the sizes of the $P_{i j}$ so that $\left|P_{i j}\right| \geq 2$ for each $i, j$ and distinct $\approx_{W}$-classes of $W$ of size at least two all have different sizes, with size at most $m+1 \in \mathbb{N}$; the number $K$ was chosen sufficiently large for this to be possible, so $K \geq \frac{(m+1)(m+2)}{2}-1$, but due to a later application of the Pigeonhole Principle $K$ will be chosen larger than this. Now every $\approx_{W}$-class of $W$ of size greater than 1 consists of a set $P_{i j}$, possibly together with an element of $U$. We will say that a set $Y \subseteq X$ is huge if $|Y|>m+1$.

Any automorphism of $(W, R)$ will preserve $\approx_{W}$, and will fix setwise each $\approx_{W^{-}}$ class of size at least two (as these classes all have different sizes). Hence, if no element of $U$ is $\approx_{W}$-equivalent to any element of any $P_{i j}$ (that is, elements of $U$ lie in $\approx_{W^{-}}$classes of size 1 ), then as the $P_{i j}$ separate the elements of $U$, any automorphism of 
$W$ will fix $U$ pointwise, as required. So the concern is that some $\approx_{W}$-class $C$ in $W$ of size at least two might consist of a set $P_{i j}$ together with some $u \in U$. In such a case there would be an automorphism of $(W, R)$ mapping $u$ to some vertex in $C \backslash\{u\}$, indeed, as observed above, any permutation of $C$, extended by the identity on $W \backslash C$, gives an automorphism of $(W, R)$.

So suppose $u \in C \cap U$ as in the last paragraph. By the Pigeonhole Principle (retaining all the above reductions, so initially working with larger sets $P_{i j}$ ) we may suppose for all such $C, u$ that either $u \| c$ (that is, $u$ and $c$ are incomparable with respect to $<$ ) for all $c \in C \backslash\{u\}$, or $u<c$ for all $c \in C \backslash\{u\}$, or $c<u$ for all $c \in C \backslash\{u\}$. For such $u, c$ and $C$, we add a finite set $S_{c u}$ of additional vertices of $\Gamma$ to $W$ according to the following recipe.

If $C$ is null, then for each $c \in C \backslash\{u\}$ for which $u \nless c$, the set $\Gamma(c) \backslash \Gamma(u)$ is infinite, and we choose infinite $S_{c u}^{\prime \prime} \subset \Gamma(c) \backslash(\Gamma(u) \cup W)$. If $C$ is complete, then for each $c \in C \backslash\{u\}$ for which $c \nless u$, the set $\Gamma(u) \backslash \Gamma(c)$ is infinite, and we choose infinite $S_{c u}^{\prime \prime} \subset \Gamma(u) \backslash(\Gamma(c) \cup W)$. In other cases $(C$ null and $u<c$ for all $c \in C \backslash\{u\}$, or $C$ complete and $c<u$ for all $c \in C \backslash\{u\})$ we do not add any corresponding set $S_{c u}^{\prime \prime}$. The sets $S_{c u}^{\prime \prime}$ are chosen so that if $(c, u) \neq\left(c^{\prime}, u^{\prime}\right)$ then $S_{c u}^{\prime \prime} \cap S_{c^{\prime} u^{\prime}}^{\prime \prime}=\emptyset$. We may suppose, again by the Pigeonhole Principle, that for each such $c, u$, either $c \| x$ for all $x \in S_{c u}^{\prime \prime}$, or $c<x$ for all $x \in S_{c u}^{\prime \prime}$, or $x<c$ for all $x \in S_{c u}^{\prime \prime}$. By Lemma 1.6 with respect to $L=\{R,<\}$, we may replace each set $S_{c u}^{\prime \prime}$ by a very large finite subset $S_{c u}^{\prime}$ in such a way that the collection of all such sets $S_{c u}^{\prime}$ is mutually indiscernible over $W$. Let $V^{\prime}$ be the union of all such sets $S_{c u}^{\prime}$ and of $W$. Observe that each $S_{c u}^{\prime}$ carries a complete or null induced subgraph of $\Gamma$, and for each $x, y \in S_{c u}^{\prime}$ and $z \in V^{\prime} \backslash S_{c u}^{\prime}$, we have $x \sim z$ if and only if $y \sim z$. In particular, any two elements of a set $S_{c u}^{\prime}$ are $\approx_{V^{\prime}}$ equivalent. Finally, since the $S_{c u}^{\prime}$ could be chosen as large (finite) as needed, we may choose huge subsets $S_{c u} \subset S_{c u}^{\prime}$ for all such $c$, $u$, so that the following holds, where $V$ is the union of $W$ and the $S_{c u}$ : all elements of $V \backslash W$ lie in huge $\approx_{V}$-classes (which may meet $W$ ), and distinct huge $\approx_{V}$-classes have different sizes. It follows that each huge $\approx_{V}$-class is fixed setwise by any automorphism of $(V, R)$.

We aim to show that every automorphism of $(V, R,<)$ must fix $U$ pointwise, which will complete the proof of the lemma. As a first step, observe that every huge $\approx_{V}$-class $S$ contains some set $S_{c u}$. We claim that no huge $\approx_{V}$-class meets $U$. For suppose $S$ is a huge $\approx_{V}$-class, with $a \in U \cap S$. There is $u \in U$ and $c \in W \backslash U$, and a $\approx_{W}$-class $C$ with $c, u \in C$, such that $S \supset S_{c u}$. Clearly $a=u$, since otherwise, as $S_{c u}$ separates $c$ from $u$, also $a$ would separate $c$ and $u$ and lie in $U \subset W$, contradicting that $c \approx_{W} u$. Now if $C$ is null then, by our rule for the process adding $S_{c u}$, all vertices of $S \backslash\{u\}$ are adjacent to $c$; hence $c$ separates $u$ from other elements of $S$, so $u \notin S$, a contradiction. Likewise, if $C$ is complete, then all vertices of $S \backslash\{u\}$ are non-adjacent to $c$, so again $c$ separates $u$ from the rest of $S$, so $u \notin S$. This proves the claim.

Claim Let $g \in \operatorname{Aut}(V, R,<)$. Then there is $h \in \operatorname{Aut}(V, R,<)_{(U)}$ such that gh fixes $W$ setwise.

Proof of Claim There are distinct (so different-sized) huge $\approx_{V}$-classes $S_{j}$ (for $j \in J$ ), each fixed setwise by $g$, such that $V \backslash W \subseteq \bigcup_{j \in J} S_{j}$. We may assume that $W$ is not fixed setwise by $g$, as otherwise the claim is trivial. Hence, for some $j \in J$, we have $\left(S_{j} \cap(V \backslash W)\right)^{g} \neq S_{j} \cap(V \backslash W)$. 
First, we show that $\left|S_{j} \cap W\right|=1$. There are $u \in U$, and some $\approx_{W}$-class $C$ containing distinct elements $u, c$ of $W$, such that $S_{j} \supseteq S_{c u}$. We may suppose that $C$ is null, and $S_{c u} \subset \Gamma(c) \backslash \Gamma(u)$, as the other case where $C$ is complete and $S_{c u} \subset \Gamma(u) \backslash \Gamma(c)$ is similar. Now no element of $W \backslash\{u, c\}$ could lie in $S_{j}$, for otherwise it would separate $u$ from $c$ in $W$ contradicting that $u \approx_{W} c$. Hence, since $S_{j}$ is a huge $\approx_{V}$-class so is disjoint from $U$, we have $S_{j} \cap W \subseteq\{c\}$. Thus, due to the existence of the element $g$, we have $S_{j} \cap W=\{c\}$. In fact, $S_{j}=S_{c u} \cup\{c\}$ : for if $c^{\prime} \in C \backslash\{u, c\}$ then $S_{c^{\prime} u} \neq \varnothing$ but $c^{\prime}$ separates elements of $S_{c^{\prime} u}$ from $c$ so elements of $S_{c^{\prime} u}$ do not lie in $S_{j}$; and if $u^{\prime} \in U \backslash\{u\}$, then no set of form $S_{d u^{\prime}}$ could be a subset of $S_{j}$, for otherwise $c$ (in $W$ ) would separate $d$ from $u^{\prime}$ so the set $S_{d u^{\prime}}$ would not have been added.

By our assumption, there is $v \in S_{c u}$ such that $v^{g}=c$. It is not possible that $S_{c u}$ is totally ordered by $<$; this follows easily from the facts that $g$ induces an automorphism of $\left(S_{c u} \cup\{c\},<\right)$, and the earlier assumption that either $c<x$ for all $x \in S_{c u}$, or $x<c$ for all $x \in S_{c u}$, or $c \| x$ for all $x \in S_{c u}$. It follows by Lemma 1.6(ii) that any permutation of $S_{c u}$, extended by the identity on the rest of $V$, is an automorphism of $(V, R,<)$. In particular distinct elements of $S_{c u}$ are <-incomparable, so as $v^{g}=c$, the set $S_{j}$ is an antichain with respect to $<$. Now it could not happen that there is some $t \in V \backslash S_{j}$ whose <-relation to $c$ is different from its <-relation to all other elements of $S_{j}$. For otherwise $t^{g^{-1}}$ would have a different $<$-relation to $v$ and to all other elements of $S_{j}$, contradicting the mutual indiscernibility in the construction of $S_{c u}$. It follows that if $g^{\prime}$ is the inverse of $g$ on $S_{j}$ and the identity on the rest of $V$, then $g^{\prime} \in \operatorname{Aut}(V, R,<)$. The element $h$ of the claim will be a product of elements of the form $g^{\prime}$, each acting on a different huge $\approx_{V}$-class.

To finish the proof of the lemma, let $g \in \operatorname{Aut}(V, R,<)$, and let $h$ be as in the claim. We must show $u^{g}=u$ for all $u \in U$. Now by construction $g h$ fixes $W$ setwise, and we claim that $g h$ fixes $U$ setwise. Indeed, suppose for a contradiction that $u \in U$ and $u^{g h} \notin U$. As the $\approx_{W}$-classes of $W$ of size greater than one are all of different sizes, they are all fixed setwise by $g h$. Hence, as all elements of $W \backslash U$ lie in $\approx_{W^{-}}$ classes of size greater than one, $u^{g h}$ and hence also $u$ lie in some $\approx_{W}$-class $C$ of size greater than one. Now, by the construction of $V$ from $U$, either $u$ is the greatest or least element of $C$ with respect to $<$, or $u$ and $u^{g h}$ are separated by some huge set of form $S_{u, u^{g h}}$. The first case is impossible as $g h$ preserves $<$. The second case is also impossible, since as the huge $\approx_{V}$-classes all have different sizes, they are fixed setwise by $g h$. Thus, as claimed, $g h$ induces an automorphism of $(W, R,<)$ which fixes $U$ setwise. Hence $g h$ fixes $U$ pointwise; for any two distinct elements of $U$ are separated by an $\approx_{W}$-class of size greater than one, and all such classes have different sizes, so are fixed setwise by $g h$. Thus, $g$ fixes $U$ pointwise.

Remark 2.4 Careful inspection of the above proof shows that if $|U|=n$, then $V$ may be chosen to have size at most $O\left(n^{8}\right)$. For in constructing $W$ from $U$, if $m=\left(\begin{array}{l}n \\ 2\end{array}\right)$ we add at most $m$ sets $P_{i j}$, each of size at least 2 and all of different sizes, so $|W|=n+k$ where $k:=|W \backslash U| \leq \frac{(m+1)(m+2)}{2}-1$. Then in adding the sets $S_{c u}$ to obtain $V$, we add at most $k$ such sets, each of size at least $m+2$, and all of different sizes. Thus, $|V \backslash W| \leq(m+2)+(m+3)+\cdots+(m+k+1)=\frac{k}{2}(2 m+k+3)$. Thus, $|V| \leq \frac{1}{2}(2 n+k(2 m+k+5))$. This is used in Theorem 4.1 below. 
Proof of Proposition 2.1 By Lemma 2.2, there is a $G$-invariant graph $\Gamma$ on $X$ such that for all distinct $x, y \in X$, the set $\Gamma(x) \triangle \Gamma(y)$ is infinite. The partial order < defined in Lemma 2.3 is clearly also $G$-invariant. The proposition thus follows immediately from that lemma.

\section{G 2-homogeneous but not 2-transitive}

By Proposition 2.1, to complete the proof of Theorem 1.1 it suffices to prove the following.

Proposition 3.1 Let $G$ be a 2-homogeneous but not 2-transitive permutation group on an infinite set $X$. Then the action of $G$ on $X$ is locally rigid.

Recall that a tournament is a directed loopless digraph $(T, \rightarrow)$ such that for any distinct vertices $x, y$, exactly one of $x \rightarrow y$ or $y \rightarrow x$ holds. A group which is 2homogeneous but not 2-transitive has just one orbit on unordered 2-sets, but two orbits on ordered pairs of distinct elements. Each of these orbits is the arc set of a $G$ invariant tournament with vertex set $X$. Thus, to prove Proposition 3.1, we develop analogues of the methods of Sect. 2, but for tournaments.

Let $\rightarrow$ denote the arc relation in a tournament $T=(X, \rightarrow)$, and let $G=\operatorname{Aut}(T)$. For $x \in X$, we let $\Gamma^{+}(x):=\{y \in X: x \rightarrow y\}$, the set of outneighbours of $x$. For $x, y, z \in X$, we say that $z$ separates $x, y$ if $x \rightarrow z \rightarrow y$ or $y \rightarrow z \rightarrow x$. Furthermore $Z \subset X$ separates $x, y$ if each $z \in Z$ separates $x, y$. We write $x \rightarrow Z$ if $x \rightarrow z$ for each $z \in Z$. We say that a tournament is rigid if it admits no non-identity automorphisms.

Proposition 3.2 Let $T=(X, \rightarrow)$ be an infinite tournament such that for any distinct $x, y \in X$, the sets $\Gamma^{+}(x) \backslash \Gamma^{+}(y)$ and $\Gamma^{+}(y) \backslash \Gamma^{+}(x)$ are both infinite. Then $T$ is cofinally rigid.

We first isolate an easy lemma, used to prove Proposition 3.2, in case it has other uses. It may be known.

Let $T=(X, \rightarrow)$ be a tournament. We will say that $A \subset X$ is a nice set if $A \neq \emptyset$ and for all $a_{1}, a_{2} \in A$ and $v \in X \backslash A$, we have $a_{1} \rightarrow v$ if and only if $a_{2} \rightarrow v$. (That is, all vertices in a nice set are related in the same way to vertices outside the nice set; equivalently, no vertex outside a nice set separates a pair of vertices inside the nice set.) Note that vacuously any singleton is a nice set, and $X$ is nice. Furthermore, we will say that $A \subset X$ is a good set, if $A$ is totally ordered by $\rightarrow$ and is nice. We consider the maximal good subsets of $X$, that is, good sets $A$ such that there is no good set $A^{\prime} \subset X$ with $A^{\prime} \supset A$.

Lemma 3.3 If $T=(X, \rightarrow)$ is a tournament, then the maximal good subsets of $X$ form a partition of $X$.

Proof We claim that if $A$ is good and $B \neq A$ is maximal good (where $A, B \subseteq X$ ), then either $A \subset B$ or $A \cap B=\emptyset$. To see this, let $d \in A \cap B$, and let $C=A \cup B$. We show that $C$ is good, which ensures $B=C$. 
Let $c_{1}, c_{2} \in C, v \in X \backslash C$. Now $c_{1} \rightarrow v$ if and only if $d \rightarrow v$ if and only if $c_{2} \rightarrow v$. This holds because $A$ and $B$ are both nice and $d \in A \cap B$. Hence $C$ is nice. If $C$ is not totally ordered, then there is some 3 -cycle $c_{1} \rightarrow c_{2} \rightarrow c_{3} \rightarrow c_{1}$ in $C$. Since $A$ and $B$ are both totally ordered, we must have at least one of these points in $A \backslash B$ and one in $B \backslash A$. Suppose $c_{1} \in A \backslash B$ and $c_{2} \in B \backslash A$ (the other case is similar). Then if $c_{3} \in A$, then $c_{2}$ separates $c_{1}, c_{3}$, contradicting the fact that $A$ is nice. Otherwise $c_{3} \in B$, then similarly $c_{1}$ separates $c_{2}, c_{3}$, contradicting the fact that $B$ is nice. Hence $C$ is totally ordered. Now $B \subseteq C$, and $C$ is good, so $A \subseteq B=C$ by maximality of $B$.

Observe also that if $\left(A_{i}\right)_{i \in I}$ is an increasing sequence of good sets (that is, $I$ is totally ordered and $A_{i} \subseteq A_{j}$ whenever $i<j$ ) then $A:=\bigcup_{i \in I} A_{i}$ is good: indeed, if either clause in the definition of good is violated then it would be violated in some $A_{i}$. Also, each singleton in $X$ is a good set. The lemma now follows immediately from the claim, using Zorn's Lemma if $X$ is infinite.

Proof of Proposition 3.2 Let $U=\left\{u_{1}, \ldots, u_{n}\right\} \subset X$. For any distinct $i, j \in\{1, \ldots, n\}$, the set $\Gamma^{+}\left(u_{i}\right) \backslash \Gamma^{+}\left(u_{j}\right)=\left\{v \in X: u_{i} \rightarrow v \rightarrow u_{j}\right\}$ is infinite. Hence by Ramsey's Theorem (indeed, a simple case of Lemma 1.6), there is $U_{i j} \subseteq \Gamma^{+}\left(u_{i}\right) \backslash\left(\Gamma^{+}\left(u_{j}\right) \cup\right.$ $\left.\left\{u_{j}\right\}\right)$ with $\left|U_{i j}\right|=\aleph_{0}$, such that $U_{i j}$ is totally ordered by $\rightarrow$. Note that the sets $U_{i j}, U_{j i}$ both separate $u_{i}, u_{j}$ ( since $u_{i} \rightarrow U_{i j} \rightarrow u_{j}$, and $u_{j} \rightarrow U_{j i} \rightarrow u_{i}$ ). We may choose the $U_{i j}$ so that if $(i, j) \neq(k, l)$ then $U_{i j} \cap U_{k l}=\emptyset$.

Claim 1 Let $N$ be any positive integer. Then there are finite subsets $V_{i j}$ of $U_{i j}$ (for all distinct integers $i, j$ with $1 \leq i, j \leq n$ ) of size $N$ such that the following holds, where $T^{\prime}$ is the induced subtournament of $T$ with vertex set $U \cup \bigcup_{i \neq j} V_{i j}$ : for any distinct $i, j \in\{1, \ldots, n\}$, and for each $x, y \in V_{i j}$ and $v \in T^{\prime} \backslash V_{i j}$, we have $x \rightarrow v$ if and only if $y \rightarrow v$.

Proof of Claim 1 This is an immediate application of Lemma 1.6.

Provided we initially choose $N$ large enough, we may cut the $V_{i j}$ down further, and so suppose that each set $V_{i j}$ has size exactly $2^{r}$ for some $r \geq 2$, and that distinct sets $V_{i j}$ and $V_{k l}$ have distinct sizes. Observe (for use in Theorem 4.1) that $T^{\prime}$ has $n+\sum_{i=2}^{m+1} 2^{i}$ vertices where $m=2\left(\begin{array}{c}n \\ 2\end{array}\right)$, that is, it has $n+2^{n^{2}-n+2}-2$ vertices. We claim that $T^{\prime}$ is rigid, which suffices to prove the lemma. Let $V$ denote the vertex set of $T^{\prime}$ (a union of $U$ and the sets $V_{i j}$ ).

The sets $V_{i j}$ are clearly all good, though possibly not maximal good. Hence, by Lemma 3.3, if $B \cap V_{i j} \neq \emptyset$ and $B$ is maximal good, then $V_{i j} \subseteq B$.

The idea of the proof is as follows. First observe that automorphisms of the subtournament $(V, \rightarrow)$ of $T$ preserve the family of maximal good sets. We aim to show that by our construction of $V$, all non-singleton maximal good sets in $V$ have different sizes, so in fact each is fixed setwise, and hence pointwise, by any automorphism. We then show that if some automorphism $\alpha$ of $(V, \rightarrow)$ fixes pointwise all non-singleton maximal good subsets of $V$, then $\alpha$ fixes $V$ pointwise.

Claim 2 If $A$ is a good subset of $V$, then $|A \cap U| \leq 1$. 
Proof of Claim 2 Suppose $u_{1}, u_{2} \in A \cap U$, with $u_{1} \neq u_{2}$. We have $u_{1} \rightarrow V_{12} \rightarrow u_{2}$. Since $A$ is good, we must have $V_{12} \subset A$ : otherwise any $y \in V_{12} \backslash A$ separates $u_{1}, u_{2}$, contradicting the fact that $A$ is nice. Similarly, we have $u_{2} \rightarrow V_{21} \rightarrow u_{1}$, and we must have $V_{21} \subset A$. But then we have $\left\{u_{1}, u_{2}\right\} \cup V_{12} \cup V_{21} \subseteq A$, and $u_{1} \rightarrow V_{12} \rightarrow u_{2} \rightarrow$ $V_{21} \rightarrow u_{1}$. But then $A$ is not totally ordered by $\rightarrow$, which contradicts the fact that $A$ is good.

Thus, maximal good sets are unions of sets $V_{i j}$ with at most one element of $U$ added (this includes the case of a singleton point of $U$ ). Then by our choice of the sizes of the $V_{i j}$ in the construction, any two non-singleton maximal good sets have different sizes. (For let the $V_{i j}$ have sizes $n_{1}, \ldots, n_{t}$, say. These were chosen as distinct powers of 2 , and so all numbers of the form $n_{i_{1}}+\cdots+n_{i_{s}}$ or $n_{i_{1}}+\cdots+n_{i_{s}}+1$ are distinct.) Hence any automorphism of $V$ fixes each non-singleton maximal good set setwise, and hence also pointwise since each is totally ordered and so rigid. Thus any automorphism fixes all elements of $V \backslash U$ pointwise, and so also fixes $U$ pointwise; indeed, for each pair of elements of $U$ there is some $Z \subset V \backslash U$ separating the pair, and so no automorphism can move points of $U$.

Corollary 3.4 Let $T$ be an infinite tournament with 2-homogeneous automorphism group. Then $T$ is cofinally rigid.

Proof By Ramsey's Theorem, there is a subtournament of $T$ of the form $\left\{x_{i}: i \in \mathbb{N}\right\}$ with $x_{i} \rightarrow x_{j}$ if and only if $i<j$ (or possibly with all arcs reversed). Clearly, if $i<j$, then $\left|\Gamma^{+}\left(x_{j}\right) \triangle \Gamma^{+}\left(x_{i}\right)\right| \geq j-i-1$. By 2-homogeneity of $\operatorname{Aut}(T)$, there is $d \in \mathbb{N} \cup\left\{\aleph_{0}\right\}$ such that if $x \neq y$ then $\left|\Gamma^{+}(x) \triangle \Gamma^{+}(y)\right|=d$. Hence, $d \geq n$ for each $n \in \mathbb{N}$, so $d=\aleph_{0}$.

We may suppose that $T$ is not totally ordered by $\rightarrow$; for otherwise, if $U$ is a finite set of vertices then the induced subtournament on $U$ is totally ordered and so rigid, and hence $T$ is cofinally rigid (as in the definition of cofinal rigidity in the Introduction we may put $V=U$ ). By Proposition 3.2, the proof of the corollary now reduces to the following claim.

Claim For all distinct $x, y \in X$, the sets $\Gamma^{+}(x) \backslash \Gamma^{+}(y)$ and $\Gamma^{+}(y) \backslash \Gamma^{+}(x)$ are both infinite.

Proof of Claim Suppose that for some $u, v \in X$ with $u \neq v$, the set $\Gamma^{+}(u) \backslash \Gamma^{+}(v)$ is infinite, but $\Gamma^{+}(v) \backslash \Gamma^{+}(u)$ is finite. Now, using 2-homogeneity, define an order relation $<$ on $X$, such that $x<y$ if and only if $\Gamma^{+}(x) \backslash \Gamma^{+}(y)$ is infinite. This is a Aut $(T)$-invariant partial order on $X$, containing comparable pairs. By 2-homogeneity, it follows that $<$ is a total order, and it or its reverse agrees with $\rightarrow$. This contradicts the above assumption.

Proof of Proposition 3.1 As noted above, there is a $G$-invariant tournament $T$ with vertex set $X$, whose arc set is a $G$-orbit on $\left\{\left(x_{1}, x_{x}\right) \in X^{2}: x_{1} \neq x_{2}\right\}$. The proposition now follows immediately from 2-homogeneity and Corollary 3.4. 
Proof of Theorem 1.1 This is immediate from Lemma 1.3 and Propositions 2.1 and 3.1.

\section{Further remarks}

The proof of Theorem 1.1 yields the following, where, for any positive integer $n, X^{[n]}$ denotes the set of $n$-element subsets of $X$ : there is a function $f: \mathbb{N} \rightarrow \mathbb{N}$ such that for any $l \in \mathbb{N}$, if $H \leq G$ are closed permutation groups on an infinite set $X$ with $G$ primitive but not 2-transitive, and $G$ and $H$ have the same orbits on $X^{[n]}$ for all $n \leq$ $f(l)$, then $G$ and $H$ have the same orbits on $X^{m}$ for all $m \leq l$. An upper bound for $f$ is given by the cardinality of $V$ in terms of $|U|$ in the definition in the Introduction of a group $G$ acting locally rigidly. By the proofs of Propositions 2.1 and 3.1, we obtain the following slight strengthening of Theorem 1.1, probably far from best possible. Observe that with $m$ and $k$ as in Remark 2.4, $\frac{1}{2}(2 n+k(2 m+k+5)) \leq n+2^{n^{2}-n+2}-2$ for all $n>1$, so the bound in the proof of Proposition 3.1 dominates.

Theorem 4.1 Let $G, H$ be closed permutation groups on the infinite set $X$, with $G$ primitive but not 2-transitive on $X$, and with $H \leq G$. Let $n \in \mathbb{N}$, and suppose that $G$ and $H$ have the same orbits on the set $X^{[l]}$ for each $l \leq n+2^{n^{2}-n+2}-2$. Then $G$ and $H$ have the same orbits on $X^{m}$ for each $m \leq n$.

Theorem 1.1 requires the assumption of primitivity. For example, $\operatorname{Aut}(\mathbb{Q},<) \mathrm{Wr} C_{2}$ is orbit-equivalent to $\operatorname{Sym}(\mathbb{Q}) \mathrm{WrC}_{2}$ (in the natural imprimitive action). However, a proof of Conjecture 1.2 should yield a lot of information about the imprimitive case.

A proof of Conjecture 1.2, at least if via local rigidity, would appear to require arguments considerably more involved than those of this paper. As an example, suppose that $G$ is 2-primitive (that is, 2-transitive and with primitive point stabilisers) but not 3-homogeneous on the infinite set $X$. We conjecture that $G$ acts locally rigidly. There is a $G$-invariant 3-hypergraph $\Gamma$ on $X$, and we would like to show that $\Gamma$ (possibly expanded by some other $G$-invariant relations) is locally rigid. Given $x \in X$, there is an induced graph $\Gamma_{x}$ on $X \backslash\{x\}$ on which $G_{x}$ acts primitively. However, it is not clear that local rigidity of $\Gamma_{x}$ transfers to local rigidity of $\Gamma$, or more generally that a straightforward induction on the degree of transitivity of $G$ can be made to work. There may also be an approach to local rigidity of hypergraphs using $[15$, Lemma 2.5] and related results.

We cannot even prove the conjecture under the assumptions that $X$ is countable and $G$ is locally compact (that is, there is some finite $F \subset X$ such that all orbits of $G_{(F)}$ on $X$ are finite). Even the case when $G$ is countable is open. A first class to consider would be that of primitive groups with finite point stabiliser, for which Smith [23] gives a useful-looking version of the O'Nan-Scott Theorem.

However, as evidence for the conjecture, we observe that a potential source of counterexamples, suggested by the family of closed supergroups of $\operatorname{Aut}(\mathbb{Q},<)$ listed in Conjecture 1.2, fails. Indeed, let $(T,<)$ be any of the countable 2-homogeneous trees (that is, semilinear orders) classified by Droste in [6]. There is a family of interesting primitive closed permutation groups associated with $\operatorname{Aut}(T,<)$, namely the 
primitive Jordan permutation groups with primitive Jordan sets classified in [1]: we have in mind $\operatorname{Aut}(T,<)$, the automorphism group of the ternary general betweenness relation on $T$ induced from $<$, the automorphism group of the corresponding countable $C$-structure, a structure whose elements are a dense set of maximal chains in $(T,<)$, and the automorphism group of the corresponding $D$-relation (a quaternary relation on the set of 'directions' of the betweenness relation). It can be checked that each of these groups acts locally rigidly. We omit the details.

One referee asked whether, in Theorem 1.1, the assumption that $H \leq G$ can be replaced by the assumption that $H$ is closed, orbit-equivalent to $G$, and not 2-transitive. The problem here is that we cannot apply the theorem to the group $G$ as a subgroup of the (topological) closure of $\langle G, H\rangle$, as the latter may be 2-transitive; and our proof of Theorem 1.1 depends essentially on Lemma 1.3, which requires the assumption $H \leq G$. In fact, as the following example shows, the referee's question has a negative answer.

Example 4.2 Let $<_{1}$ be the natural order on $\mathbb{Q}$, and let $<_{2}$ be defined on $\mathbb{Q}$ by putting $x<2 y$ if $y<_{1} \pi<_{1} x$, or if $x<_{1} y$ and $x, y$ both lie in $\mathbb{Q} \cap(\pi, \infty)$ or both in $\mathbb{Q} \cap(-\infty, \pi)$. Put $G=\operatorname{Aut}\left(\mathbb{Q},<_{1}\right)$ and $H:=\operatorname{Aut}\left(\mathbb{Q},<_{2}\right)$. Then $G, H$ are distinct 2-homogeneous but not 2-transitive closed groups which are each orbit-equivalent to $\operatorname{Sym}(\mathbb{Q})$, and so are orbit-equivalent to each other. In fact the topological closure of $\langle G, H\rangle$ is the (2-transitive) automorphism group of the natural circular order on $\mathbb{Q}$.

Possibly this is essentially the only such example: in any such examples $G, H$ must be 2-homogeneous, not 2-transitive permutation groups on $X$ such that the two non-diagonal $G$-orbits on $X^{2}$ are not the same as those of $H$. So we modify the referee's question, and ask the following.

Question 4.3 Is there an example of a pair of distinct orbit-equivalent primitive but not 2-transitive closed permutation groups $G$ and $H$ on an infinite set $X$, such that at least one of $G, H$ does not preserve a total order on $X$ ?

In [5] a permutation group $G$ on $X$ is defined to be orbit-closed if there is no $H \leq \operatorname{Sym}(X)$ which properly contains $G$ and is orbit-equivalent to $G$. Such $G$ will be a closed permutation group, and Conjecture 1.2 asserts that if $X$ is countably infinite then the only primitive closed permutation groups which are not orbit-closed are the proper subgroups of $\operatorname{Sym}(X)$ listed in that conjecture. In [5] the authors define $G \leq \operatorname{Sym}(X)$ to be a relation group if there is a collection $R$ of finite subsets of $X$ such that

$$
G=\left\{g \in \operatorname{Sym}(X): \forall a \in \mathcal{P}(X)\left(a \in R \leftrightarrow a^{g} \in R\right)\right\} .
$$

Clearly any relation group is orbit-closed. Also, by [5, Corollary 4.3], any finite primitive orbit-closed group is a relation group. We do not know whether this holds without finiteness, and in particular cannot answer the following question, to which Siemons drew our attention.

Question 4.4 Is $\operatorname{Aut}(\mathbb{Q},<)$ the only primitive but not 2-transitive closed permutation group of countable degree which is not a relation group? 
As a small example, let $\Gamma_{3}$ be the universal homogeneous 2-edge-coloured graph with edges coloured randomly red or green; that is, the unique countably infinite homogeneous 2-edge-coloured graph such that for any three finite disjoint sets $U, V, W$ of vertices, there is a vertex $x$ not adjacent to any vertex in $U$, adjacent by a red edge to each element of $V$ and by a green edge to each element of $W$. At first sight, $G=\operatorname{Aut}\left(\Gamma_{3}\right)$ is not a relation group, but in fact it is a relation group; for we may take $R$ to consist of the 2 -sets joined by a red edge and the 3 -sets which carry a green triangle.

Our remarks in the Introduction suggest a further question. Again, for convenience, we shall consider actions on a countably infinite set $X$. A subset $Y$ of $X$ is a moiety of $X$ if $|Y|=|X \backslash Y|$.

Question 4.5 Which primitive closed permutation groups $G$ on a countably infinite set $X$ have a regular orbit on moieties?

To say that $G$ has a regular orbit on moieties of $X$ is close to the condition, in the language of $[14,24]$ and elsewhere, that any first order structure $M$ on $X$ with $G=\operatorname{Aut}(M)$ has distinguishing number 2, that is, that $G$ has a regular orbit on the power set $\mathcal{P}(X)$. Some results on these conditions are obtained in [14]. For example, if $M$ is a homogeneous structure such that the collection of finite structures which embed in it is a 'free amalgamation class', and $\operatorname{Aut}(M)$ is primitive but for some $k$ is not $k$-transitive, then $\operatorname{Aut}(M)$ has a regular orbit on moieties, and so distinguishing number 2. In particular, this holds for the random graph, as follows already from [11, Theorem 3.1]. On the other hand, as noted in [14] it is easily seen that $\operatorname{Aut}(\mathbb{Q},<)$ has no regular orbit on $\mathcal{P}(\mathbb{Q})$. Indeed, clearly there is no regular orbit on finite sets; and if $A$ is a moiety of $\mathbb{Q}$ whose setwise stabiliser is trivial, then $A$ is dense and codense in $\mathbb{Q}$, but the structure $(\mathbb{Q},<, P)$, where $P$ is a unary predicate naming a dense codense set, is homogeneous so admits $2^{\aleph_{0}}$ automorphisms.

This suggests the following strengthening of orbit-equivalence. Recall from the Introduction the notion of strong orbit equivalence. The following conjecture is implied by Conjecture 1.2, for it is easily seen that the five closed groups containing $\operatorname{Aut}(\mathbb{Q},<)$ all have different orbits on $\mathcal{P}(\mathbb{Q})$. For example, $\operatorname{Aut}(\mathbb{Q},<)$ has an orbit consisting of increasing subsets of order type $\omega$ with rational supremum, but this family of sets is not invariant under the automorphism groups of the induced circular order or linear betweenness relation.

Conjecture 4.6 Let $G, H$ be strongly orbit-equivalent closed permutation groups on the countably infinite set $X$. Then $H=G$.

Again, the assumption that the groups are closed is necessary. Stoller ([25], see also [17]) gives an example of a proper subgroup $H$ of $G=\operatorname{Sym}(\mathbb{N})$ which is strongly orbit-equivalent to $G$; namely, let $H$ consist of those permutations $g$ of $\mathbb{N}$ such that there are two partitions, dependent on $g$, of $\mathbb{N}$ into finitely many sets $A_{1}, \ldots, A_{k}$ and $B_{1}, \ldots, B_{k}$ (so $\mathbb{N}=A_{1} \cup \cdots \cup A_{k}=B_{1} \cup \cdots \cup B_{k}$, each partitions) such that for each $i=1, \ldots, k$, the element $g$ induces an order isomorphism $\left(A_{i},<\right) \rightarrow\left(B_{i},<\right)$.

Finally, we mention a conjectural strengthening of Lemmas 1.4 and 2.3. It is a special case of a much stronger conjecture in [7]. 
Conjecture 4.7 Let $\Gamma$ be an infinite graph such that for any distinct vertices $x, y$ the set $\Gamma(x) \triangle \Gamma(y)$ is infinite. Then $\Gamma$ is locally rigid.

Acknowledgement We thank J. Siemons for drawing attention to [5] and [23] and to Question 4.4. We also thank both referees for careful reports, from which the presentation has benefitted. This research was supported by EPSRC grant EP/H00677X/1.

\section{References}

1. Adeleke, S., Neumann, P.M.: Primitive permutation groups with primitive Jordan sets. J. Lond. Math. Soc. 53, 209-229 (1996)

2. Cameron, P.J.: Transitivity of permutation groups on unordered sets. Math. Z. 148, 127-139 (1976)

3. Cameron, P.J.: Oligomorphic Permutation Groups. London Math. Soc. Lecture Notes, vol. 152. Cambridge University Press, Cambridge (1990)

4. Cameron, P.J., Neumann, P.M., Saxl, J.: On groups with no regular orbits on the set of subsets. Arch. Math. 43, 295-296 (1984)

5. Dalla Volta, F., Siemons, J.: Orbit equivalence and permutation groups defined by unordered relations. J. Algebr. Comb. 35, 547-564 (2012)

6. Droste, M.: Structure of Partially Ordered Sets with Transitive Automorphism Groups. Mem. Amer. Math. Soc., vol. 334. Amer. Math. Soc., Providence (1985)

7. Droste, M., Giraudet, M., Macpherson, H.D., Sauer, N.: Set-homogeneous graphs. J. Comb. Theory, Ser. B 62, 63-95 (1994)

8. Enomoto, H.: Combinatorially homogeneous graphs. J. Comb. Theory, Ser. B 30, 215-223 (1981)

9. Fraïssé, R.: Theory of Relations. Studia Logic Found. Math., vol. 145. North-Holland, Amsterdam (2000). Revised edn., with an appendix by N. Sauer

10. Gray, R., Macpherson, H.D., Praeger, C.E., Royle, G.F.: Set-homogeneous directed graphs. J. Comb. Theory, Ser. B 102, 474-520 (2012)

11. Henson, W.: A family of countable graphs. Pac. J. Math. 38, 69-83 (1971)

12. Inglis, N.: On orbit equivalent permutation groups. Arch. Math. 43, 297-300 (1984)

13. Lachlan, A.H.: Finite homogeneous simple digraphs. In: Stern, J. (ed.) Logic Colloquium 1981. Studia Logic Found. Math., vol. 107, pp. 189-208. North-Holland, New York (1982)

14. Laflamme, C., Nguyen van Thé, L., Sauer, N.: Distinguishing number of countable homogeneous relational structures. Electron. J. Comb. 17, R20 (2010)

15. Macpherson, H.D.: The action of an infinite permutation group on the unordered subsets of a set. Proc. Lond. Math. Soc. 46, 471-486 (1983)

16. Macpherson, H.D.: Orbits of infinite permutation groups. Proc. Lond. Math. Soc. 51, 246-284 (1985)

17. Neumann, P.M.: Homogeneity of infinite permutation groups. Bull. Lond. Math. Soc. 20, 305-312 (1988)

18. Ronse, C.: On homogeneous graphs. J. Lond. Math. Soc. 17, 375-379 (1978)

19. Seress, A.: Primitive groups with no regular orbits on the set of subsets. Bull. Lond. Math. Soc. 29, 697-704 (1997)

20. Seress, A., Yang, K.: On orbit-equivalent two-step imprimitive permutation groups. In: Computational Group Theory and the Theory of Groups. Contemp. Math., vol. 470, pp. 271-286. Amer. Math. Soc., Providence (2008)

21. Siemons, J.: On partitions and permutation groups on unordered sets. Arch. Math. 38, 391-403 (1982)

22. Siemons, J., Wagner, A.: On finite permutation groups with the same orbits on unordered sets. Arch. Math. 45, 492-500 (1985)

23. Smith, S.M.: A classification of primitive permutation groups with finite stabilizers. arXiv:1109. 5432v1 [math.GR]

24. Smith, S.M., Tucker, T.W., Watkins, M.E.: Distinguishability of infinite groups and graphs. Electron. J. Comb. 19, P27 (2012)

25. Stoller, G.: Example of a subgroup of $S_{\infty}$ which has a set-transitive property. Bull. Am. Math. Soc. 69, 220-221 (1963) 